\title{
Enhanced dc magnetic field response based on a piezoelectric unimorph and an energized coil
}

\author{
Wei He, Gaofeng Che, Tzong-Jer Chen \\ School of Information Engineering, Baise University, Baise 533000, China \\ *weiheky@yeah.net
}

Keywords: piezoelectric unimorph; energized coil; Ampere force; magnetic field sensor

\begin{abstract}
In this paper, a device is developed for detecting external dc magnetic field, which is constructed from a piezoelectric unimorph and an energized coil. The working principle of the device is based on the coupling of the Ampere force in the energized coil exposure to external magnetic field and the piezoelectric effect of the piezoelectric unimorph. Experiments have been conducted to verify the feasibility of the device. The device shows a high output voltage of $0.5984 \mathrm{~V}$ at the dc magnetic field of $0.1 \mathrm{mT}$. The large dc magnetic field response of the proposed structure driven by the Ampere force makes this device hopeful in application of dc magnetic field sensors.
\end{abstract}

\section{Introduction}

Magnetoelectric laminate composites, which consists of magnetostrictive and piezoelectric layers, have been widely studied duo to their large response to external magnetic field [1]-[7]. Compared with single phase materials, the composites have larger magnetoelectric coefficient. Typically, the composites fabricated from giant magnetostrictive materials (e.g., Terfenol-D) and piezoelectric materials can attain a strong magnetoelectric response to external magnetic field due to the large magnetostriction of Terfenol-D [8], [9]. However, the magnetoelectric effect of the laminate composites is mainly concerned with external ac magnetic field, and they are not suited for dc or quasi-dc magnetic field [10], [11].

This paper presents a dc magnetic field detector to overcome the issue of the magnetoelectric laminate composites. The principle of the proposed device is based on the moment effect resulting Ampere forces and piezoelectric effect of the piezoelectric unimorph. The experimental results validate the presented design. The features of low-cost, simplicity, and large response make the proposed device promising in real applications.

\section{Structure and principle}

Fig. 1 shows the schematic diagram of the proposed structure. The device consists of a piezoelectric unimorph, a coil, and two fixed blocks. When the coil is energized, an Ampere force is induced on the upper block, which is given by

$$
F=n \mu_{0} H_{d c} I L,
$$

where $n$ is the turns of the coil, $\mu_{0}$ is the space permeability, $H_{\mathrm{dc}}$ is the external magnetic field, $I$ is the current, and $L$ is the length of the coil on top surface of the upper block. The Ampere force on the surface of the nether fixed block is equal in magnitude and opposite in direction to the force $F$, as shown in Fig. 1. The piezoelectric unimorph vibrates under the Ampere forces. The piezoelectric material works in $d_{31}$ mode, and the piezoelectric equations can be given by [12]

$$
\begin{aligned}
& S_{1}=s_{11}^{D} T_{1}+g_{31} D_{3}, \\
& E_{3}=\frac{1}{\varepsilon_{33}^{T}} D_{3}-g_{31} T_{1},
\end{aligned}
$$

where $S_{1}$ and $T_{1}$ are respectively the strain and stress in the piezoelectric material, $s_{11}^{D}$ denotes the elastic compliance coefficient, $g_{31}$ is the piezoelectric voltage constant, and $E_{3}$ and $D_{3}$ represent the 
electric field strength and electric displacement, respectively. Assuming $D_{3}=0$ (open circuit), the voltage across the top and bottom surfaces of the piezoelectric material can be expressed as

$$
V_{3}=E_{3} d=\frac{-g_{31} S_{1}}{s_{11}^{D}}
$$

where $\mathrm{d}$ is the thickness of the piezoelectric material. From Eq. 4, it can be seen that the output voltage $V_{3}$ is proportional to the stress $S_{1}$, which is induced by the Ampere forces according to Eq. 1 .

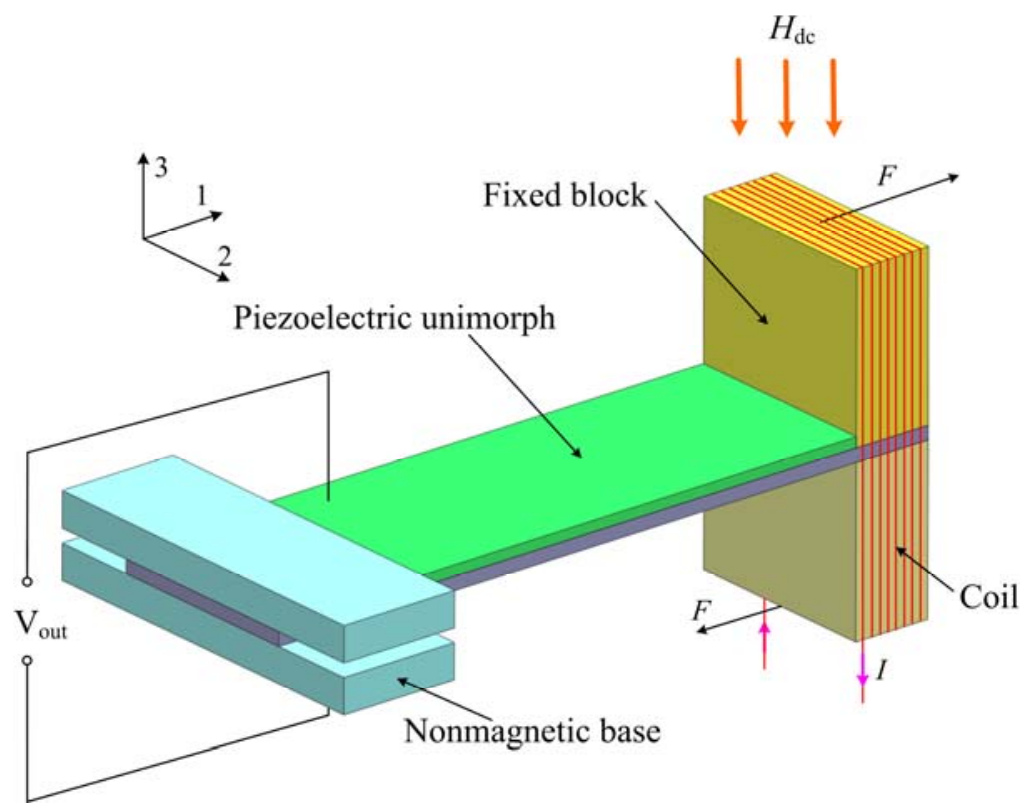

Fig. 1. Schematic diagram of the proposed structure

\section{Results and discussions}

A prototype is fabrication according to Fig. 1. The piezoelectric unimorph consists a piezoelectric layer and a substrate layer. The two layers of the unimorph are bonded using adhesive under load. The material of the fixed blocks is aluminum. The output voltage of the device is measured by an oscilloscope.

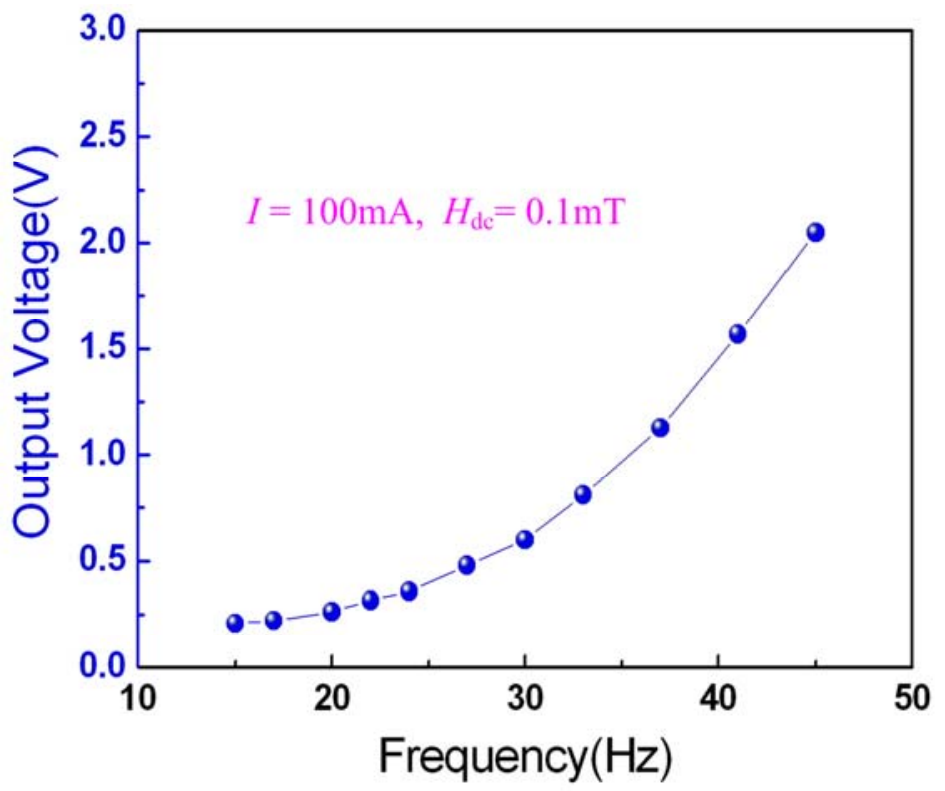

Fig. 2. Output voltage as a function of frequency at $0.1 \mathrm{mT}$ 
The external dc magnetic field is set at $0.1 \mathrm{mT}$, and the current in the coil is $100 \mathrm{~mA}$. Fig. 2 shows the output voltage versus frequency of the electric current under non-resonant state. It can be seen from Fig. 2 that, the voltage increases with the exciting frequency. When the frequency varies from $10 \mathrm{~Hz}$ to $45 \mathrm{~Hz}$, the voltage is increased from $0.209 \mathrm{~V}$ to $2.048 \mathrm{~V}$. For instance, at the frequency of 30 $\mathrm{Hz}$, an output voltage of $0.5984 \mathrm{~V}$ can be obtained at the dc magnetic field of $0.1 \mathrm{mT}$. The strong response of the device to external magnetic field is due to the Ampere forces and the resulting moments.

\section{Conclusions}

In this paper, we have developed a dc magnetic detector based on Ampere forces. Under an applied external dc magnetic field and an ac electric current in the coil, resulting moments are induced, and the piezoelectric unimorph vibrates and generates voltage output. The strong response makes the device ergastic for detecting dc or quasi-dc magnetic field.

\section{Acknowledgment}

This work is supported by the National Natural Science Foundation of China (Grant No. 61540039) and the Natural Science Foundation of Guangxi Province (Grant No. 2015GXNSFBA139263).

\section{References}

[1] Ryu J, Priya S, Carazo A V, et al. Effect of the Magnetostrictive Layer on Magnetoelectric Properties in Lead Zirconate Titanate/Terfenol - D Laminate Composites[J]. Journal of the American Ceramic Society, 2001, 84(12): 2905-2908.

[2] Wen Y, Wang D, Li P. Enhanced giant magnetoelectric effect in laminate composites of FeCuNbSiB/FeNi/PZT [J]. Journal of Magnetics, 2011, 16(4): 398-402.

[3] Lu C, Li P, Wen Y, et al. Investigation of magnetostrictive/piezoelectric multilayer composite with a giant zero-biased magnetoelectric effect [J]. Applied Physics A, 2013, 113(2): 413-421.

[4] Chen L, Li P, Wen Y, et al. Analysis of the low-frequency magnetoelectric performance in three-phase laminate composites with Fe-based nanocrystalline ribbon [J]. Smart Materials and Structures, 2013, 22(11): 115031.

[5] Zhai J, Xing Z, Dong S, et al. Magnetoelectric laminate composites: an overview [J]. Journal of the American Ceramic Society, 2008, 91(2): 351-358.

[6] Park C S, Cho K H, Arat M A, et al. High magnetic field sensitivity in $\mathrm{Pb}(\mathrm{Zr}, \mathrm{Ti}) \mathrm{O}_{3}-\mathrm{Pb}$ $\left(\mathrm{Mg}_{1 / 3} \mathrm{Nb}_{2 / 3}\right) \mathrm{O}_{3}$ single crystal/Terfenol-D/Metglas magnetoelectric laminate composites [J]. Journal of Applied Physics, 2010, 107(9): 094109.

[7] Zhou H M, Li C, Xuan L M, et al. Equivalent circuit method research of resonant magnetoelectric characteristic in magnetoelectric laminate composites using nonlinear magnetostrictive constitutive model [J]. Smart Materials and Structures, 2011, 20(3): 035001.

[8] Dong S X, Cheng J R, Li J F, et al. Enhanced magnetoelectric effects in laminate composites of Terfenol-D/Pb (Zr, Ti) $\mathrm{O}_{3}$ under resonant drive [J]. Applied Physics Letters, 2003, 83(23):4812-4814.

[9] Dong S X, Li J F, Viehland D. A longitudinal-longitudinal mode Terfenol-D/ $\mathrm{Pb}\left(\mathrm{Mg}_{1 / 3} \mathrm{Nb}_{2 / 3}\right) \mathrm{O}_{3}-\mathrm{PbTiO}_{3}$ laminate composite [J]. Applied Physics Letters, 2004, 85(22):5305-5306.

[10] Leung C M, Or S W, Ho S L. dc magnetoelectric sensor based on direct coupling of Lorentz force effect in aluminum strip with transverse piezoelectric effect in $0.7 \mathrm{~Pb}\left(\mathrm{Mg}_{1 / 3} \mathrm{Nb}_{2 / 3}\right)$ $\mathrm{O}_{3}-0.3 \mathrm{PbTiO}_{3}$ single-crystal plate[J]. Journal of Applied Physics, 2010, 107(9): 09E702. 
[11] Jia Y, Tang Y, Zhao X, et al. Additional dc magnetic field response of magnetostrictive /piezoelectric magnetoelectric laminates by Lorentz force effect [J]. Journal of Applied Physics, 2006, 100(12): 126102.

[12] T. Ikeda, Fundamentals of Piezoelectricity, Oxford University Press, Oxford, 1990. 